\title{
Los impactos de la dinámica del mercado de trabajo sobre la actividad económica en Puerto Rico
}

\author{
Wilfredo Toledo* \\ wilfredotoledo@aol.com \\ Econometrics and Stat Research, \\ San Juan, Puerto Rico
}

\section{RESUMEN}

El objetivo de este artículo fue determinar la importancia relativa de cambios inesperados en la oferta y la demanda de trabajo sobre la trayectoria del empleo en una economía con estancamiento en el mercado laboral. Para esos propósitos se calibró un modelo de equilibrio general de ciclos económicos reales con datos de Puerto Rico. Los resultados de ese modelo revelaron que los impulsos de las preferencias por ocio pueden tener un efecto importante en las fluctuaciones del insumo laboral. Además, se estimaron dos modelos SVAR utilizando restricciones de signos en uno de ellos y restricciones de largo plazo en otro, para identificar las perturbaciones mencionadas. Las estimaciones de esos modelos sugieren que los impulsos de la demanda de trabajo (productividad y/o choques de la inversión) explican entre 50 y 70 por ciento de las desviaciones del empleo en la isla de su tendencia de crecimiento. También implican que los impulsos de la oferta de trabajo son responsables de al menos $30 \%$ de las fluctuaciones en el empleo de la isla en el largo plazo. Por tanto, aun en economías con altas tasas de desempleo existe la posibilidad de estimular el empleo, mejorando las compensaciones y las condiciones de trabajo de los empleados para promover la participación laboral, además de las políticas convencionales del lado de la demanda de trabajo.

Palabras clave: modelo de equilibrio general dinámico y estocástico; impulsos de la oferta y demanda de trabajo; modelo svar con restricciones de signos; Puerto Rico

\footnotetext{
${ }^{*}$ Investigador/Consultor, doctor en economía y estadístico profesional acreditado (PStat ${ }^{\circledR}$ ) por la American Statistical Association.
} 


\title{
The impacts of labor market dynamics on economic activity in Puerto Rico
}

\begin{abstract}
This article seeks to determine the importance of labor supply and demand shocks on employment dynamics in an economy with a stagnant labor market. A DSGE model was calibrated using data from Puerto Rico. The simulation of this model revealed that preference shocks to leisure might significantly impact cyclical variations of the labor input. Further evidence was obtained by imposing sign and long-run restrictions on two SVAR models to identify the aforementioned disturbances. The estimation of these models showed that labor demand disturbances accounted for 50 percent to 70 percent of employment fluctuations in the island. It was also found that labor supply shocks explained at least 30 percent of the forecast error variance of the labor input. Hence, even in economies with high unemployment rates, there is a possibility of stimulating employment by raising workers' compensation and improving job quality to promote labor force participation, besides the traditional labor demand policies.
\end{abstract}

Keywords: dsge model; labor supply and demand shocks; svar model with sign restrictions; Puerto Rico

JEL Codes: C32, E24, E32

Artículo recibido: 20 diciembre. 2021 Aceptado para publicación: 10 enero 2022 Correspondencia: wilfredotoledo@aol.com Conflictos de Interés: Ninguna que declarar 


\section{INTRODUCCIÓN}

La actividad económica no siempre determina el comportamiento del empleo a través del ciclo económico. En ocasiones las empresas no reducen el número de empleados ante perturbaciones agregadas que reducen la demanda por sus productos. La existencia de costos asociados al despido y contratación de empleados y expectativas de una pronta recuperación son algunas de las razones que se han expuesto para explicar esas decisiones de las firmas. Si las empresas subestiman la duración de la recesión pudieran mantener trabajadores en exceso para evitar los costos mencionados.

También, se ha observado que el desempleo no se reduce rápidamente cuando la economía se mueve del régimen de recesión al de expansión. La explicación del párrafo anterior puede servir como una causa de este fenómeno: si las empresas mantuvieron un número de empleados superior al óptimo en los períodos de contracción económica, entonces al expandir la producción incrementan la productividad de estos y no su nivel. Esto es, reducen el tiempo de ocio que los trabadores disfrutan en sus horas laborables. Sin embargo, las transferencias públicas que reciben, a través de distintos programas sociales, los trabajadores por períodos prolongados de tiempo también pueden conducir a ese comportamiento observado en las series de empleo y desempleo.

En los artículos sobre este tema desde el punto de vista microeconómico se modelan las decisiones de los individuos con respecto a su participación en el mercado laboral. Por otro lado, las investigaciones que examinan el asunto con datos agregados utilizan principalmente la tasa de desempleo como el indicador de la situación del mercado del trabajo. En este artículo se utiliza un enfoque agregado, pero se examina el comportamiento del empleo y los salarios como las variables principales de interés. Algunos autores se concentran en los factores del lado de la oferta de trabajo, mientras que otros examinan aquellos que impactan la demanda de ese insumo de producción. En esta investigación se examinan los impactos sobre el empleo de perturbaciones de la demanda y oferta de trabajo. En el estudio se utilizan datos de Puerto Rico, que un es un país que ha manifestado altas tasas de desempleo y bajas tasas de participación laboral por varias décadas. En ese país, por ejemplo, los promedios de la tasa de participación, la tasa de empleo y la tasa de desempleo, de 2015 a 2019, fueron de cerca 40, 36 y 11 por ciento, respectivamente (véase Cuadro 1). Por lo que la isla resulta ser un buen ejemplo de economías con estancamiento en el mercado laboral. Para realizar el análisis 
del tema se utilizó un modelo estocástico dinámico de equilibrio (DSGE, por sus siglas en el inglés) y la estimación de dos modelos autorregresivos estructurales (SVAR): un modelo principal y otro que se utiliza para examinar la sensibilidad de los resultados del primero con respecto a las restricciones impuestas y a la omisión de variables.

El examen de la dinámica del mercado de trabajo es importante para el diseño de políticas económicas anticíclicas. En la delineación de esas políticas no siempre se debe tener como objetivo final reducir el desempleo, sino que pudiera, en algunas instancias, ser más importante enfocarse en un indicador de la oferta de trabajo. como la tasa de participación. Por lo que es relevante determinar la importancia relativa de las perturbaciones de la oferta y demanda de trabajo en el desempeño del mercado laboral. Los resultados de esta investigación pueden servir como base para el desarrollo de medidas de políticas públicas dirigidas a mejorar la situación del mercado de trabajo en economías con estancamiento económico.

\begin{tabular}{|c|c|c|c|}
\hline \multicolumn{4}{|c|}{$\begin{array}{l}\text { Cuadro } 1 \\
\text { Estadísticas Descriptivas de Tres Indicadores del Mercado de Trabajo en Puerto Rico } \\
\text { ( Porcentajes, 2015-2019) }\end{array}$} \\
\hline & Tasa de Participación & Tasa de Empleo & Tasa de Desempleo \\
\hline Media & 40.08 & 35.67 & 10.98 \\
\hline Mediana & 40.10 & 35.54 & 11.50 \\
\hline Valor Máximo & 40.60 & 37.10 & 12.80 \\
\hline Valor Mínimo & 39.60 & 34.54 & 8.50 \\
\hline $\begin{array}{l}\text { Número de } \\
\text { Observaciones }\end{array}$ & 5 & 5 & 5 \\
\hline
\end{tabular}

La organización del resto del artículo es como sigue. La próxima sección se dedica a la exposición de una muestra de los trabajos sobre el tema estudiado. La sección 3 presenta un modelo de ciclos económicos reales que puede ser utilizado para determinar algunos aspectos de los efectos de distintas perturbaciones sobre el empleo y otras variables que definen el estado de la economía. La metodología de la investigación está contenida en la sección 4, mientras que en la 5 se examinan los resultados de una simulación del modelo de equilibrio general. En la sección 6 se analizan los hallazgos de la estimación de los modelos SVAR, y la última sección contiene las conclusiones de la investigación. 


\section{REVISIÓN BIBLIOGRÁFICA}

En esta sección se exponen los resultados de los artículos más relacionados con este estudio. Primero se discuten investigaciones que proveen explicaciones teóricas para el estancamiento observado en las variables que definen el mercado laboral, y más adelante se presentan trabajos con contenidos empíricos. Clark y Summers (1982) plantean que en Estados Unidos la tasa de desempleo exhibe una tendencia de largo plazo ascendente. De acuerdo a esos autores, algunas posibles explicaciones para ese comportamiento son: las transferencias por seguro de desempleo, reducción en la intensidad del proceso de búsqueda de empleo a través del tiempo, la existencia de salarios de reserva altos y la no disposición de aceptar ofertas de empleo con salarios bajos, y la provisión de información falsa por parte de los encuestados (estos pudieran aseverar falsamente que están buscando empleo para obtener los beneficios dirigidos a los desempleados, lo que incrementa la tasa de desempleo). De estos factores, ellos hacen énfasis en el rol de los beneficios que reciben los desempleados en algunos países como uno de los principales determinantes de la decisión de buscar y dejar empleos. La posibilidad de calificar para los pagos del seguro por desempleo atrae a algunas personas a incorporarse a la fuerza laboral, con el objetivo ulterior de abandonarla una vez cumplan con los requisitos para recibir la compensación por desempleo, plantean Clark y Summers. En teoría, entonces, los pagos a los desempleados pueden aumentar la fuerza laboral y la tasa de desempleo.

El problema del desempleo creciente y persistente (histéresis) que Europa Occidental ha experimentado desde los mil novecientos setenta es analizado por Blanchard y Summers (1986). Ese fenómeno, indican ellos, es muy difícil de explicar utilizando tanto las teorías clásicas como las keynesianas. Rigideces en salarios y precios, como en los modelos keynesianos, pudieran ser causa de altos niveles de desempleo, pero es muy poco probable que puedan explicar su persistencia. Por otro lado, argumentan los autores que la sustitución intertemporal en el ocio, como aparece en los modelos de los nuevos clásicos, no parece tampoco arrojar luz sobre el origen de esa situación. Aseveran Blanchard y Summers que la hipótesis del club pudiera ser útil para analizar la alta autocorrelación que muestra la serie de desempleo. En ese contexto se pudiera pensar en los empleados como los miembros del club (insiders) y los desempleados (outsiders) los que no pertenecen al mismo. En el proceso de establecer los salarios participan sólo los empleados no los desempleados (que necesitan empleos) por lo que existe una asimetría: 
los que no son miembros del club pudieran estar en la disposición de aceptar salarios más bajos, para salir del desempleo, que los que reciben los miembros, pero no tienen poder en la toma de esas decisiones. Por lo que se pudiera establecer un nivel de salario que no les permita ser reclutados.

Los cambios estructurales que expanden ciertos sectores económicos y contraen otros pueden generar parte del desempleo observado en los Estados Unidos, señala Summers (1986). La especialización del recurso humano hace que su movimiento entre sectores sea costoso. Por ejemplo, los individuos que pierden empleos de alta calidad esperan obtener ese mismo tipo de puesto de trabajo y no aceptan salarios bajos. Esos salarios altos pueden explicarse bajo la teoría de salarios eficientes: las empresas mantienen salarios altos -en cierto recorrido- incluso si hay exceso de oferta laboral, porque tal acción incrementa la productividad del trabajo, aumenta la moral de los empleados y puede reducir su rotación, por lo que disminuyen los costos asociados a despidos y contrataciones. Así que, los empleados pudiesen atrapar parte de las ganancias de las empresas. Además, un factor que no menciona el autor, pero que puede promover la persistencia del desempleo en este tipo de trabajadores, son los hábitos de consumo y los compromisos económicos contraídos. Los individuos que pierden salarios altos pueden tener gastos fijos elevados como hipotecas, préstamos de automóviles y otros, que no pueden satisfacer con salarios bajos, por lo que les puede tomar mucho tiempo en obtener una oferta de empleo aceptable. También, como menciona Summers, el que los individuos accedan a recibir salarios bajos pudiera ser interpretado como falta de competencia o baja productividad. En base a este análisis se puede conjeturar que perturbaciones sectoriales que impactan sectores con salarios altos pueden ser responsables de cambios en la relación vacantes-desempleo, porque algunos desempleados no están dispuestos a trabajar por salarios inferiores a los que recibían antes.

En una línea un poco distinta Goettle et at. (2008) afirman que la oferta laboral depende principalmente de factores demográficos y tecnológicos. De acuerdo con esos autores cuando se reduce el número de las personas en la edad de trabajo, la pérdida se compensa parcialmente con incrementos continuos en la calidad del trabajo o en la productividad. Ese aumento en la productividad pudiera ser el resultado de reclutar personas con niveles mayores de educación, reduciendo el tiempo de ocio que los individuos disfrutan en las 
horas laborables, o utilizando una nueva tecnología que utilice menos insumo laboral. Autor (2007), amplía ese punto al mencionar que existe un tipo de tecnología que es segada hacia ciertas destrezas de los trabajadores, lo que pudiera propiciar desempleo de largo plazo para ciertos tipos de ocupaciones.

Por otra parte, Uhlig (2003) afirma que la dinámica de las horas trabajadas y la productividad, además de depender de los avances en tecnología, pueden verse afectada por cambios en las actitudes sociales hacia el trabajo. Modificaciones en la estructura de impuestos sobre los ingresos provenientes del capital alteran los niveles óptimos de ese insumo y por consiguiente la productividad del trabajo y su valor de equilibrio. Por ejemplo, un incremento en ese tipo de gravamen disminuye el ahorro y aumenta los niveles de ocio y de otros bienes y por consiguiente se reducen las horas trabajadas.

Un planteamiento innovador en esta literatura, esgrimido por Uhlig, es la importancia de cambios en las visiones que tengan los individuos sobre el trabajo. Los trabajadores pudieran considerar una fracción de horas laborables como tiempo de interacción social u ocio. Ejemplo de este tipo de actividades son acceso a la internet, viajes de negocios, y uso de facilidades recreativas. Si los patronos determinan la tasa salarial en base a las horas que aportan a la producción y no de acuerdo con las horas contratadas, pudieran retener más empleados que los necesarios. Así que, adelantos tecnológicos, que incrementan la productividad del trabajo, pudieran no aumentar las horas trabajadas, sino simplemente reducir el tiempo de ocio en el empleo.

En el aspecto empírico, Galí (1999) utiliza las restricciones de largo plazo en un sistema VAR para descomponer los impulsos que afectan la productividad y el insumo laboral, en innovaciones tecnológicas y de otro tipo. Para el estudio se utilizan datos trimestrales de los Estados Unidos para el período de 1948:1 a 1994:4 y para el período de posguerra para los otros países del G-7 $7^{1}$ El autor construye dos modelos uno donde el indicador del insumo laboral son horas trabajadas y otro usando el empleo. Los dos sistemas se estiman en las primeras diferencias de las variables. Las funciones de impulso-respuesta de los sistemas estimados por dicho autor revelan que los cambios tecnológicos inesperados aumentan la productividad y reducen el trabajo. Hallazgos similares sobre el efecto negativo de los shocks tecnológicos sobre el empleo son obtenidos por Shea

\footnotetext{
${ }^{1}$ Los países son Canadá, el Reino Unido, Alemania, Francia, Italia, y Japón. El período del análisis varió para cada país por la disponibilidad de datos.
} 
(1999) Francis y Ramey (2002) y Basu, Fernald y Kimball (2006). Por otro lado, análisis realizados por Christiano, Eichenbaum y Vigfusson (2003) sugieren que los avances tecnológicos ocasionan alzas en las horas trabajadas en Estados Unidos, lo que implica que esos impulsos estimulan la demanda de trabajo. Pesavento y Rossi (2003) llegan a esa misma conclusión.

Otro trabajo significativo es el de Shapiro y Watson (1988) quienes, utilizando datos para los Estados Unidos en el período de posguerra, encuentran que los choques de la oferta de trabajo explican cerca de $60 \%$ de las variaciones en las horas trabajadas en el corto plazo y el $100 \%$ en el largo plazo. Esos resultados son compatibles con los de Foroni, Furlanetto y Lepetit (2016) que utilizan restricciones de signos, basados en un modelo nuevo keynesiano, para identificar los choques de la oferta de trabajo en un modelo de vectores autorregresivos (VAR). De acuerdo con sus análisis las perturbaciones de la oferta de trabajo explican cerca del 50\% de las variaciones en la tasa de participación laboral de Estados Unidos. En contraposición, Erceg y Levin (2014) y Aaronson et al. (2014) concluyen que la tasa de participación depende principalmente de cambios en la demanda de trabajo.

En otros artículos relevantes para el tema de esta investigación se examinan los efectos de impulsos de la demanda y oferta agregada sobre distintos indicadores que describen del estado de la economía. Las perturbaciones del lado de la oferta pueden ser el resultado de avances tecnológicos que incrementan la productividad de los insumos. Por ejemplo, Blanchard and Quah (1989), utilizando datos de Estados Unidos, de 1950 a 1987, hallan evidencia de que la tasa de desempleo experimenta alzas leves como respuesta a cambios inesperados en la oferta agregada, pero después de unos pocos trimestres, se contrae hasta que alcanza valores negativos y luego vuelve a su nivel de equilibrio. Según los autores, este hallazgo sugiere la existencia de rigideces en los salarios reales, lo que hace que los aumentos de la productividad marginal no se reflejen en los mismos y por tanto no se estimule el empleo en respuesta a los impulsos favorables de tecnología. No obstante, ellos señalan que la tasa de desempleo depende principalmente de impulsos de lado de la demanda agregada. En la misma línea Braun, Bock y DiCecio (2009), usando datos para el mismo país para el período de 1954 a 2004, plantean que existe una respuesta positiva de la demanda laboral a las perturbaciones de productividad, pero que las horas trabajadas dependen tanto de impulsos de la oferta como de la demanda agregada. 


\section{UN MODELO TEÓRICO}

En esta sección se presenta una descripción de una economía donde se puede examinar el tema planteado en este artículo. Para esos propósitos se utiliza una modificación de un modelo simple ampliamente utilizado en la literatura de los ciclos económicos reales ${ }^{2}$, según presentado por Collard (2009), al que se le añade una perturbación que afecta la oferta de trabajo. En esta economía el consumidor representativo maximiza la siguiente función de preferencias:

$$
E\left[\beta^{t}\left(\ln C_{t}-\varphi \eta_{t}\left(\frac{\left.\left(L_{t}\right)^{1+\frac{1}{v}}\right)}{1+\frac{1}{v}}\right)\right)\right]
$$

Donde; $0<\beta<1$ es el factor de descuento, $\eta_{t}$ es un proceso estocástico que impacta $\varphi>0$, un parámetro que mide la reducción en utilidad que genera el trabajo, $C_{t}$ es consumo, $L_{t}$ es trabajo, y v es la elasticidad de la oferta de trabajo de Frish.

La forma de introducir el impulso a la oferta laboral $\left(\eta_{t}\right)$ en la función de utilidad, implica que el mismo se relaciona en forma inversa con las horas de trabajo ofrecidas en el mercado. Por lo que puede ser interpretado como cualquier tipo de política o evento que desaliente el empleo o reduzca el costo del ocio. Pero también, pudiera reflejar cambios demográficos que afecten la oferta de trabajo de los hogares, o impulsos que alteren la tasa de sustitución entre ocio y consumo. La evolución de ese shock que impacta la oferta laboral se puede representar como:

$$
\begin{aligned}
& \eta_{t}=e^{\psi_{t}} \\
& \psi_{t}=\rho \psi_{t-1}+\epsilon_{L S, t}
\end{aligned}
$$

Donde $\epsilon_{t}$ es un elemento estocástico con media cero y varianza constante, $y|\rho|<1$.

Los individuos se confrontan con la siguiente restricción presupuestaria:

$$
C_{t}+i_{t}=Y_{t}
$$

\footnotetext{
${ }^{2}$ Véase, por ejemplo, McCallum (1988).
} 
Esta restricción implica que los recursos (la producción, Y) se dedican al consumo y a incrementar el capital el próximo período (i es inversión). Si $\delta$ es la tasa de depreciación, la evolución del capital está dada por:

$K_{t+1}=e^{\tau_{t}} i_{t}+(1-\delta) K_{t}$

Donde $\tau_{t}$ es un proceso estocástico que afecta la formación de capital.

En esa economía las empresas contratan el trabajo, alquilan el capital y producen los bienes finales de acuerdo con:

$Y_{t}=e^{\lambda_{t}} K^{\alpha} L^{1-\alpha}$

Siendo, $\lambda_{t}$ es un elemento estocástico de tecnología que sigue un proceso AR(1).

Los procesos estocásticos que afectan la formación de capital y la producción se suponen que están correlacionados y se describen por:

$\tau_{t}=\gamma \lambda_{t-1}+\phi \tau_{t-1}+\epsilon_{K, t}$

$\lambda_{t}=\phi \lambda_{t-1}+\epsilon_{T E C, t}$

Donde $\in_{T E C, t} y \in_{K, t}$ son elementos estocásticos con media cero y varianza constante, $y$ $|\phi| y|\gamma|<1$.

Las condiciones del primer orden del proceso de maximización del modelo y las de equilibrio constituyen el siguiente sistema de ocho ecuaciones:

$C_{t} \eta_{t} \varphi_{t}\left(L_{t}\right)^{1+\frac{1}{v}}=(1-\alpha) Y_{t}$

$1=\beta E\left[\left(\frac{e^{\tau} t C_{t}}{e^{\tau} t+1 C_{t+1}}\right)\left(e^{\tau_{t+1}} \alpha \frac{Y_{t+1}}{K_{t+1}}\right)+1-\delta\right]$

$Y_{t}=e^{\lambda_{t}} K^{\alpha} L^{1-\alpha}$

$K_{t+1}=e^{\tau_{t+1}} i_{t}+(1-\delta) K_{t}$

$\eta_{t}=e^{\psi_{t}}$

$\psi_{t}=\rho \psi_{t-1}+\epsilon_{L S, t}$

$\tau_{t}=\gamma \lambda_{t-1}+\phi \tau_{t-1}+\epsilon_{K, t}$

$\lambda_{t}=\phi \lambda_{t-1}++\gamma \tau_{t-1}+\epsilon_{T E C, t}$

Esas ecuaciones definen el estado de la economía. La primera ecuación contiene la dinámica del trabajo que se afecta directamente por la perturbación de la oferta de trabajo y a través de la producción por la de tecnología. La ecuación de Euler se define en (2) y contiene información sobre decisiones de consumo y capital (decisiones intertemporales). Las demás ecuaciones ya han sido explicadas. 


\section{METODOLOGÍA Y DATOS}

El tema planteado en este artículo se examina con dos metodologías distintas. El modelo descrito en la sección anterior se calibró con datos trimestrales de Puerto Rico y se resolvió para examinar la dinámica de las variables de interés ante perturbaciones de tecnología y de la oferta laboral. Se utilizaron los programas Dynare y Matlab para esos propósitos. Además, se estimó un modelo de vectores autorregresivo estructural (SVAR por sus siglas en inglés), utilizando datos anuales, al que se impusieron restricciones de signos.

\subsection{Especificación del Sistema SVAR}

El modelo presentado en la sección anterior parte de una economía real, donde la producción se utiliza para el consumo y los gastos de capital. Esa condición se puede modificar para un tipo de economía en que los hogares suplen la fuerza laboral a un salario (de W). Para completar el sistema es necesario identificar algún indicador del insumo laboral. En este este estudio se utiliza el empleo total, ya que no cuenta con datos de las horas trabajadas para la economía examinada. En el modelo SVAR se modelan explícitamente los impulsos de la oferta y demanda por trabajo utilizando restricciones de signos. El sistema se representa como:

$$
\left[\begin{array}{c}
\Delta L_{t} \\
\Delta(W / P) t
\end{array}\right]=\left[\begin{array}{ll}
\Phi_{11}(L) & \Phi_{12}(L) \\
\Phi_{21}(L) & \Phi_{22}(L)
\end{array}\right]\left[\begin{array}{c}
\Delta L_{t} \\
\Delta(W / P)_{t}
\end{array}\right]+\left[\begin{array}{c}
\varepsilon_{L D} \\
\varepsilon_{L S}
\end{array}\right]
$$

Donde: $L$ el empleo agregado y $(W / P)_{t}$ es el salario real, $\quad \Phi(L)=\Phi_{1} L+$ $\Phi_{2} L^{2}+\ldots+\Phi_{p} L^{P}$ es un polinomio en el operador de rezagos $L, \epsilon_{L D}$ y $\epsilon_{L S}$ son los shocks de la demanda y oferta por trabajo, respectivamente. Se incluyeron variables binarias para modelar los cambios estructurales que afectaron las series durante el período de análisis.

Al sistema (9) hay que imponerle algunas restricciones para identificar las perturbaciones de la demanda y la oferta de trabajo. Una perturbación desfavorable a la oferta de trabajo disminuye el nivel de empleo y aumenta los salarios, mientras que los adelantos de tecnología aumentan la productividad del insumo laboral estimulando su demanda, lo que ocasionan alzas en los salarios y empleos. La disparidad en la respuesta de los salarios ante los dos tipos de impulsos se puede utilizar para identificar los mismos. Por tanto, para extraer los impulsos estructurales del sistema (1) se imponen 
restricciones sobre los signos de la respuesta de las variables del sistema ante los cambios no anticipados (Faust (1998), Uhlig (2005), Canova y De Nicoló (2002) son algunos de los artículos que utilizan ese tipo de identificación). Se supone, además, que los dos tipos de innovaciones son ortogonales. El Cuadro 2 resume la identificación.

En síntesis, en esta metodología se identifican econométricamente impulsos del lado de la oferta y la demanda de trabajo. Los primeros provienen de varias fuentes como: aumentos en la preferencia por ocio, modificaciones en los pagos de transferencias a individuos y cambios en los impuestos sobre salarios. Por otra parte, las perturbaciones del lado de la demanda de trabajo están asociadas principalmente a cambios tecnológicos que incrementan la productividad del insumo laboral.

\begin{tabular}{|c|c|c|}
\hline \multicolumn{3}{|c|}{$\begin{array}{l}\text { Cuadro } 2 \\
\text { Esquema de Identificación de los Impulsos en el Sistema (9) } \\
\text { con Restricciones de Signos }\end{array}$} \\
\hline Variable & $\begin{array}{c}\text { Perturbación de } \\
\text { la oferta de trabajo } \\
\text { (Alzas en las Preferencias por Ocio) }\end{array}$ & $\begin{array}{c}\text { Perturbación de } \\
\text { la demanda de trabajo } \\
\text { (Alzas en Productividad) }\end{array}$ \\
\hline Empleo (L) & - & + \\
\hline $\begin{array}{ll}\text { Salario } & \text { real } \\
(\mathrm{W} / \mathrm{P}) & \end{array}$ & + & + \\
\hline
\end{tabular}

\subsection{Datos y Análisis Preliminares}

En esta investigación se utilizaron datos anuales de Puerto Rico para el período comprendido entre 1950 y 2019. Los datos se obtuvieron de la Junta de Planificación de Puerto Rico. Se usaron todas las observaciones disponibles de las variables. El Cuadro 3 contiene algunas estadísticas descriptivas del empleo y el salario real. Se encontró, como era anticipable, que las dos variables se mueven conjuntamente, el coeficiente de correlación de Pearson entre las misma fue 0.88. Una prueba de causalidad de Granger (utilizando dos rezagos según determinado por el criterio de información de Akaike) arrojó que el salario real causa el empleo con un nivel de significancia (Valor-P) de 0.013 cuando se usan los niveles de las series y de 0.019 al utilizar las primeras diferencias. Las estimaciones con datos de a través del tiempo requieren que las series sean estacionarias. El Cuadro 4 contiene los resultados de la prueba de Dickey-Fuller aumentada que se utilizó para examinar esa propiedad. Como es evidente, las dos series 
son $\mathrm{I}(1)^{3}$. Ese resultado se validó tomando en cuenta la tendencia determinista lineal y de segundo grado, y considerando los cambios estructurales en las series. Se realizaron pruebas de cointegración para las series y se encontró que las mismas no están cointegradas (véase Cuadro 5). Para esta prueba se consideraron tendencias lineales y de segundo grado en el modelo VAR y en las relaciones de cointegración y los resultados se mantuvieron. Estos hallazgos sugieren que la especificación del modelo (9) en las primeras diferencias de las variables es correcta.

\begin{tabular}{|l|c|c|}
\hline $\begin{array}{l}\text { Cuadro 3 } \\
\text { Estadísticas Descriptivas de las Variables Utilizadas para el Estimar el Modelo SVAR } \\
(1950-2019)\end{array}$ & Empleo en miles \\
\hline \multicolumn{1}{|c|}{ Estadístico } & $\begin{array}{c}\text { Salario real anual por empleado, } \\
\text { en dólares de 1954 }\end{array}$ \\
\hline Media & 2,896 & 846 \\
\hline Mediana & 3,096 & 766 \\
\hline Valor Máximo & 4,205 & 539 \\
\hline Valor Mínimo & 740 & \\
\hline \multicolumn{2}{|l|}{} \\
\hline
\end{tabular}

\begin{tabular}{|}
$\begin{array}{l}\text { Cuadro } 4 \\
\text { Resultados de las Pruebas de Raíces Unitarias } \\
\text { (Tendencia Determinista Lineal) }\end{array}$ \\
\hline \multicolumn{2}{|c|}{ Variable } & Estadístico-ADF & Valor-P \\
\hline Salario Real (W/P) & & 0.8916 \\
\hline Niveles & -1.247 & 0.0000 \\
\hline Primera Diferencia & -6.626 & 0.6718 \\
\hline Empleo (L) & -1.845 & 0.0085 \\
\hline Niveles & -4.148 & \\
\hline Primera Diferencia & Fuente: Elaboración propia \\
\hline
\end{tabular}

\footnotetext{
${ }^{3}$ Se llegó a la misma conclusión al utilizar la prueba de estacionariedad de Kwiatkowski-PhillipsSchmidt-Shin (KPSS test).
} 


\begin{tabular}{|c|c|c|c|c|}
\hline \multicolumn{5}{|c|}{$\begin{array}{l}\text { Cuadro } 5 \\
\text { Prueba de Cointegración de Johansen } \\
\text { (Tendencia Determinista Lineal) }\end{array}$} \\
\hline \multicolumn{5}{|c|}{ Muestra (ajustada): 1953-2019; Rezagos: 2} \\
\hline \multicolumn{5}{|c|}{ Prueba de Cointegración irrestricta (traza) } \\
\hline $\begin{array}{l}\text { Número. de relaciones de } \\
\text { cointegración en } \mathrm{H}_{0}\end{array}$ & $\begin{array}{c}\text { Valor } \\
\text { Propio }\end{array}$ & $\begin{array}{l}\text { Estadístico- } \\
\text { Traza }\end{array}$ & $\begin{array}{l}\text { Valor Critico } \\
\text { a } 0.05\end{array}$ & Valor-P* \\
\hline Ninguna & 0.112 & 9.891 & 15.494 & 0.289 \\
\hline Al menos una & 0.028 & 1.916 & 3.841 & 0.166 \\
\hline \multicolumn{5}{|c|}{$\begin{array}{l}\text { La prueba de la traza indica que no existen relaciones de cointegración a un nivel de } \\
\text { significancia de } 0.05 \text {. }\end{array}$} \\
\hline \multicolumn{5}{|c|}{ Prueba de Cointegración Irrestricta (valor propio máximo) } \\
\hline $\begin{array}{l}\text { Número de relaciones de } \\
\text { cointegración en } \mathrm{H}_{0}\end{array}$ & $\begin{array}{c}\text { Valor } \\
\text { propio }\end{array}$ & $\begin{array}{l}\text { Estadístico- } \\
\text { valor propio } \\
\text { máximo }\end{array}$ & $\begin{array}{l}\text { Valor Critico } \\
\text { a } 0.05\end{array}$ & Valor-P* \\
\hline Ninguna & 0.112 & 7.975 & 14.264 & 0.381 \\
\hline Al menos una & 0.028 & 1.916 & 3.841 & 0.166 \\
\hline \multicolumn{5}{|c|}{$\begin{array}{l}\text { La prueba del valor propio-máximo indica no cointegración a un nivel de } 0.05 \text {. * } \\
\text { Valores-P de MacKinnon-Haug-Michelis (1999). } \\
\text { Fuente: Elaboración propia }\end{array}$} \\
\hline
\end{tabular}

En la estimación del modelo SVAR se tomaron en cuenta los cambios estructurales que afectaron las series. La prueba de Quandt-Andrews fue utilizada para identificar las fechas de los quiebres. Para la calibración del modelo de equilibrio general se utilizó el promedio de los últimos cinco años de los datos de la isla y se tomaron algunos parámetros de la literatura cuando no existía información para Puerto Rico. El modelo de equilibrio general, contrario al modelo SVAR, se ajustó en frecuencia trimestral.

\section{SIMULACIÓN DEL MODELO DSGE}

En esta sección se presenta la simulación del modelo para examinar sus propiedades dinámicas. El modelo fue calibrado en frecuencia trimestral, como se mencionó. Los parámetros utilizados para calibrar el modelo se detallan en el Cuadro 6. Por otro lado, el Cuadro 7 contiene los coeficientes de autocorrelación hasta el orden cuatro para las variables generadas por el modelo. Como se observa, existe una alta correlación contemporánea entre las series principales, como sucede en las economías capitalistas. Como la variable de interés en esta investigación es el insumo laboral (L) se estimó la 
función de autocorrelación (ACF, por sus siglas en inglés) para la serie de empleo observada en Puerto Rico para el período total y un subperíodo reciente, más cercano a los años usados para calibrar el modelo, pero con la longitud necesaria para estimar la ACF. Como es evidente en el Cuadro 8, los coeficientes de autocorrelación de L hasta el tercer orden en el período de 1999-2019 son muy similares a los estimados con la serie generada en la simulación del modelo.

\begin{tabular}{|c|l|c|}
\hline $\begin{array}{l}\text { Cuadro 6 } \\
\text { Valores de los parámetros utilizados en la calibración }\end{array}$ & Valor \\
\hline Parámetro & \multicolumn{1}{|c|}{ Descripción } \\
\hline$\varphi$ & Efecto en la utilidad de la reducción en el trabajo (>0) & 2.95 \\
\hline$\alpha$ & $\begin{array}{l}\text { Exponente del capital en la función de producción (k } \\
\text { share) }\end{array}$ & 0.40 \\
\hline$\beta$ & Factor de descuento en preferencias intertemporales & 0.99 \\
\hline$\delta$ & Tasa de depreciación & 0.03 \\
\hline$\phi$ & $\begin{array}{l}\text { Persistencia de dos procesos AR(1): el de tecnología } \\
\text { que afecta Y \& el que afecta la inversión. }\end{array}$ & 0.95 \\
\hline$\gamma$ & $\begin{array}{l}\text { Persistencia cruzada del proceso de tecnología \& el } \\
\text { que afecta la inversión. }\end{array}$ & 0.025 \\
\hline$\rho$ & Persistencia de proceso que afecta la oferta de trabajo & 0.90 \\
\hline$v$ & Elasticidad de Frisch & 0.35 \\
\hline
\end{tabular}

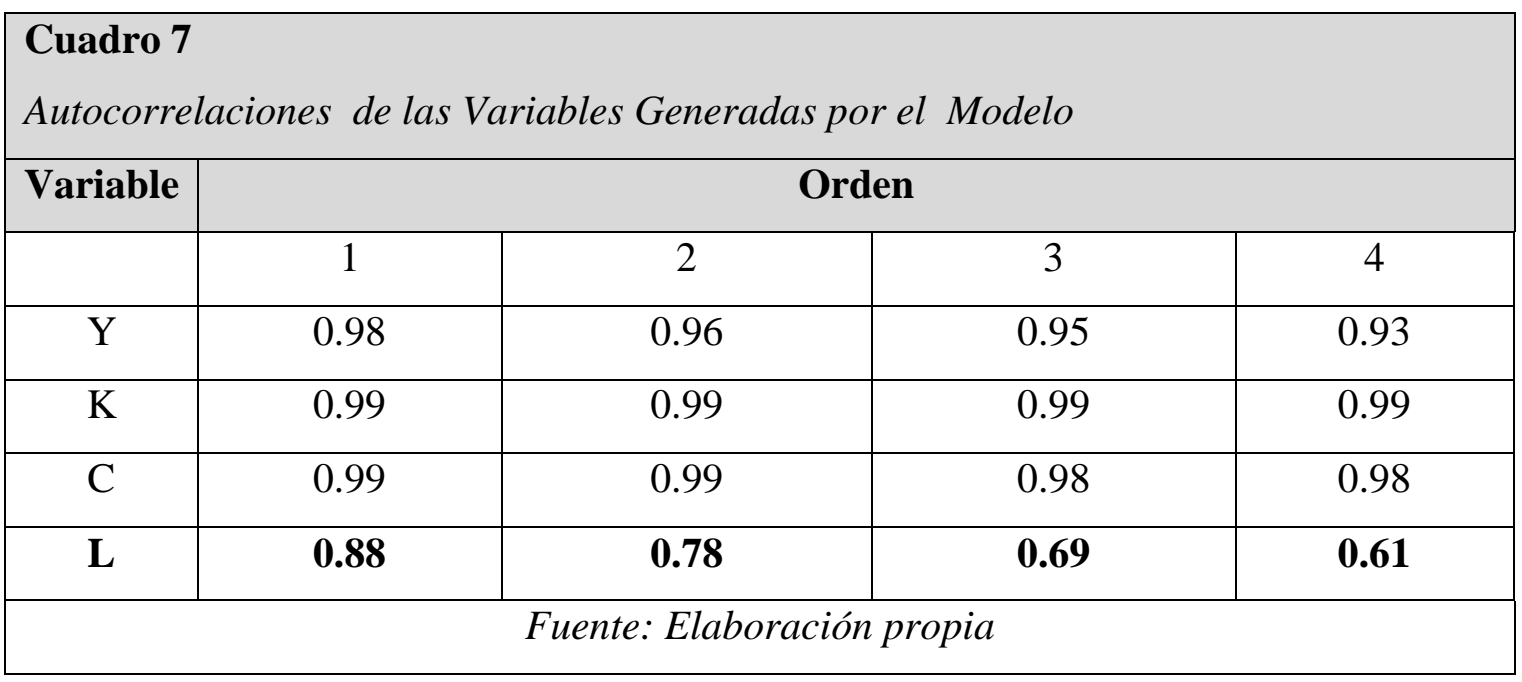




\begin{tabular}{|c|c|c|c|c|}
\hline \multicolumn{5}{|c|}{$\begin{array}{l}\text { Cuadro } 8 \\
\text { Autocorrelaciones del Empleo en Puerto Rico } \\
\text { ( Datos anuales) }\end{array}$} \\
\hline \multirow[t]{2}{*}{ Período } & \multicolumn{4}{|c|}{ Orden } \\
\hline & 1 & 2 & 3 & 4 \\
\hline $1950-2019$ & 0.98 & 0.96 & 0.93 & 0.89 \\
\hline $1999-2019$ & 0.91 & 0.77 & 0.60 & 0.41 \\
\hline \multicolumn{5}{|c|}{ Fuente: Elaboración propia } \\
\hline
\end{tabular}

Para analizar la dinámica del modelo se utilizan las funciones de impulso-respuesta y la descomposición de la varianza del error de predicción. Las funciones de impulso respuesta de Y y L se presentan en las Gráficas 1 y 2, respectivamente (las Gráficas de $\mathrm{C}$ y K no se presentan para ahorrar espacio, pero siguen el movimiento de Y). En la economía ficticia se definieron tres perturbaciones, dos que afectan el lado de la producción (una que afecta Y directamente y otra a través de la inversión) y una tercera que aumenta las preferencias por ocio. Por lo que es necesario determinar si los indicadores macroeconómicos seleccionados tienen la respuesta esperada ante esas innovaciones. Es evidente en la Gráfica 1, que la producción real se incrementa como resultado de los impulsos de tecnología que aumentan la productividad de los insumos de producción y amplían las posibilidades de consumo en esa economía. Por otro lado, Y se reduce como consecuencia del alza inesperada en las preferencias por ocio.

Las respuestas de las horas trabajadas ante los tres impulsos se muestran en la Gráfica 2. El trabajo se incrementa como resultado inmediato de los impulsos de tecnología e inversión. Sin embargo, en el largo plazo (horizonte 39 en adelante) experimenta una leve reducción. Eso podría implicar el uso más intensivo del capital por el incremento en su productividad generado por los shocks mencionados. Además, como era anticipado, las horas trabajadas merman como consecuencia del incremento en las preferencias por ocio.

El Cuadro 9 contiene los resultados de la estimación de la función de la descomposición de varianza del error de predicción. Los impulsos de tecnología y de capital parecen ser más importantes que el de la oferta de trabajo para la dinámica de la producción (Y), el capital (K) y el consumo (C). La relevancia de cambios tecnológicos inesperados en la economía de la isla ha sido señalada anteriormente por Toledo (2006) y Alemar y Rodríguez (2020). 
En contraposición la perturbación de la oferta de trabajo se le puede atribuir un impacto menor sobre Y, K y C. Sin embargo, ese tipo de shock es responsable de cerca de $43 \%$ de las fluctuaciones en las horas trabajadas. Ese porcentaje se reduce a 30\% cuando el coeficiente de AR(1) del shock en la preferencia por ocio es 0.75 en lugar de 0.90 . Lo que indica que la importancia de los impulsos a la oferta de trabajo depende críticamente de ese parámetro. Es de esperarse que estos impulsos de preferencia persistan a través del tiempo, por lo que ese coeficiente debe ser considerablemente alto.
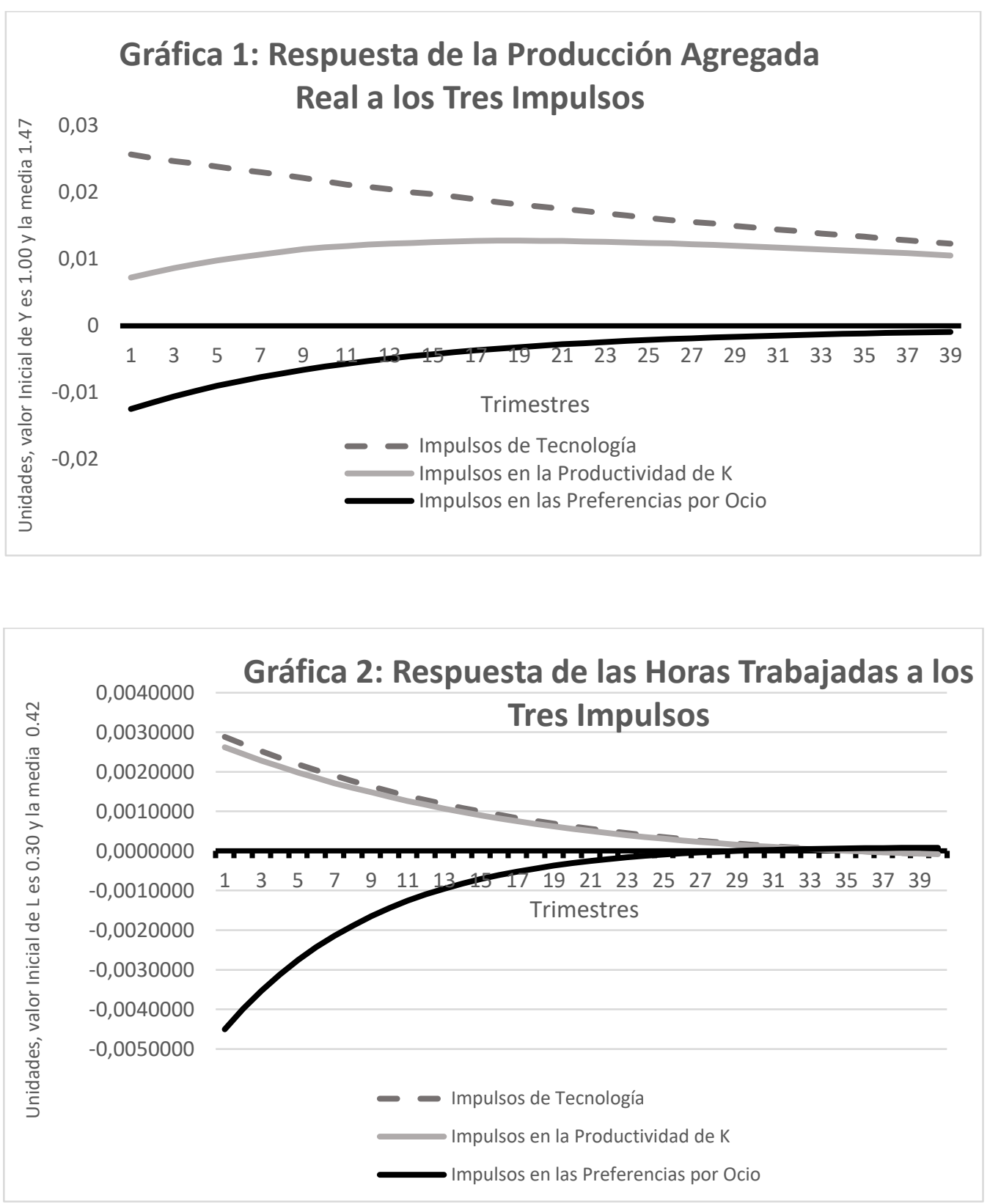
Esta simulación sugiere que pueden existir economías donde la dinámica de las horas trabajadas dependa de características del mercado laboral que afecten la valoración del consumo y el ocio. Sin embargo, en este experimento se encontró que cerca de la mitad de las fluctuaciones en $\mathrm{L}$ dependen de impulsos permanentes, asociados principalmente a la demanda de trabajo.

\begin{tabular}{|c|c|c|c|}
\hline $\begin{array}{l}\text { Cuadro } \\
\text { Descomposición de la Varianza del Error de Predicción } \\
\text { (Modelo DSGE) }\end{array}$ \\
\hline \multirow{2}{*}{ Variable } & \multicolumn{4}{|c|}{ Porcentaje de la varianza atribuible a impulsos en: } \\
\cline { 2 - 4 } & Tecnología & La productividad del capital & La oferta laboral \\
\hline Y & 62 & 24 & 5 \\
\hline C & 57 & 28 & 3 \\
\hline K & 53 & 38 & 43 \\
\hline L & 26 & 20 & 2 \\
\hline $\begin{array}{l}\text { Nota: Las cifras no suman hasta } \\
\text { choques simulados en muestras pequeñas y la no linealidad. Esta es la descomposición } \\
\text { asintótica. }\end{array}$ \\
\multicolumn{4}{|c}{ Fuente: Elaboración propia } \\
\hline
\end{tabular}

\section{RESULTADOS DE LA ESTIMACIÓN DEL MODELO SVAR}

En este modelo la identificación de los impulsos estructurales se obtuvo imponiendo la restricción principal de que alzas en las preferencias por ocio ${ }^{4}$ aumentan los salarios reales y reducen el nivel de empleo de equilibrio, ya que reducen la oferta de trabajo. Las restricciones se impusieron sólo al primer impacto de las perturbaciones utilizando el enfoque bayesiano. Las funciones de impulso-respuesta de los salarios reales y el empleo ante choques de la oferta de trabajo se presentan en la Gráfica 3 y 4 . Como es evidente, la respuesta inicial de las dos variables ante perturbación bajo análisis refleja las restricciones de signos impuestas.

Para evaluar la importancia de los impulsos de la oferta de trabajo explicando las desviaciones de las variables del sistema de su trayectoria de largo plazo se estimaron las funciones de descomposición del error de predicción (FDEP), las mismas se resumen en

\footnotetext{
${ }^{4}$ Cambios demográficos, también pueden afectar la oferta de trabajo, pero es de esperarse que impacten principalmente su tendencia de crecimiento y no sus fluctuaciones cíclicas.
} 
el Cuadro 10. Los impulsos de la oferta de trabajo explican cerca de un tercio de las fluctuaciones experimentadas por el empleo total en la isla ${ }^{5}$. Este resultado coincide con los hallazgos de Chang y Schordifheid (2003) para el caso de Estados Unidos. Su estudio concluye que $30 \%$ de las fluctuaciones en las horas trabajadas en ese país pueden explicarse por impulsos de la oferta de trabajo. Smets y Wouters (2007), también señalan la relevancia de ese tipo de shock para la dinámica de las horas de trabajo. Esa misma conclusión es alcanzada por Hall (1997), que utilizando datos de EE. UU. de 1947 a 1993, examina los efectos de cambios en las preferencias por ocio en la actividad económica. Su trabajo reveló que la tasa de sustitución de consumo por ocio es la principal fuerza que mueve las fluctuaciones económicas en ese país, en el período examinado. Ese autor, afirma que las recesiones son períodos de tiempo en que los individuos reducen el consumo de bienes y servicios y por consiguiente reducen las horas de trabajo. Foroni, Furlanetto y Lepetit (2016), por otro lado, utilizan restricciones de signos, basados en un modelo nuevo keynesiano, para identificar los choques de la oferta de trabajo en un modelo de vectores autorregresivos (VAR). Los autores informan que las perturbaciones de la oferta de trabajo explican cerca del $50 \%$ de las variaciones en la tasa de participación de Estados Unidos para el período de 1985 a 2014.

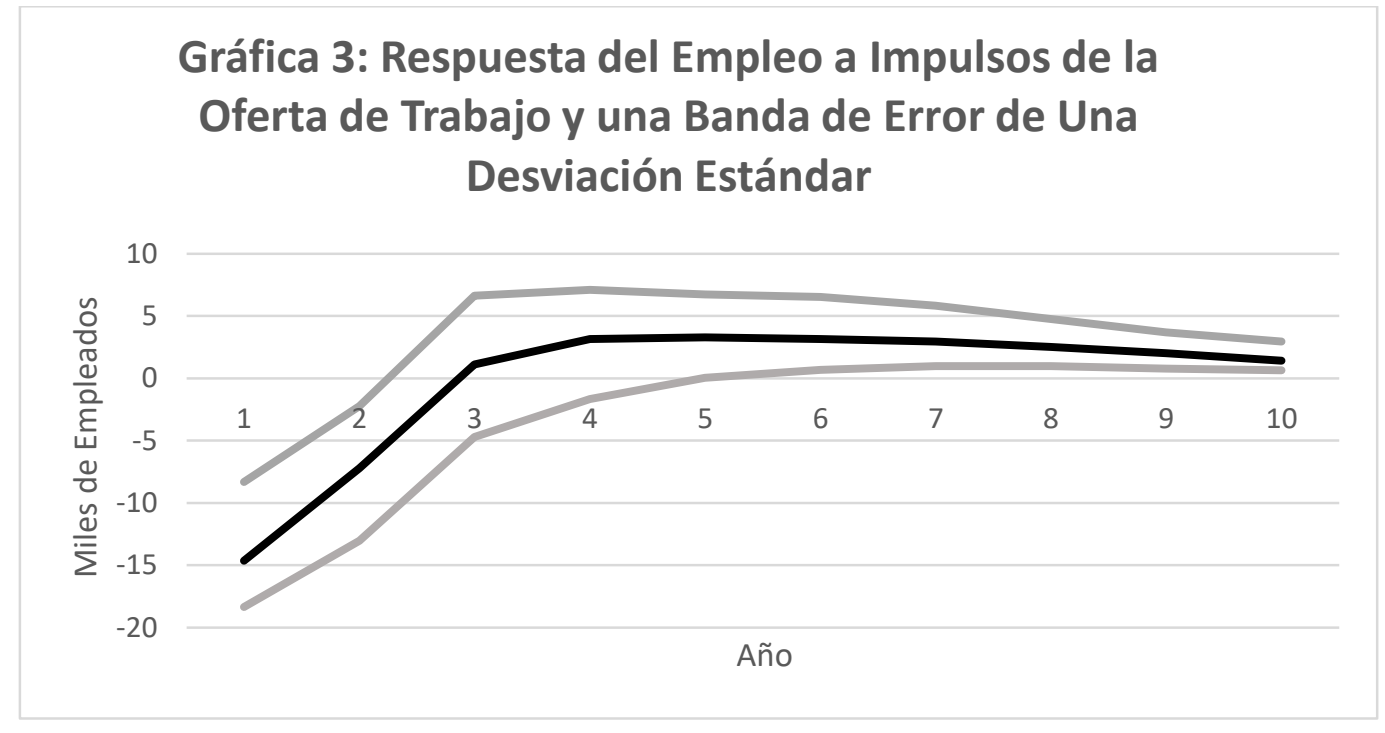

\footnotetext{
${ }^{5}$ Este resultado se mantiene cuando se sustituyen las primeras diferencias de las variables con las series filtradas para remover las tendencias deterministas.
} 


\section{Gráfica 4: Respuesta del Salario Real a Impulsos de la Oferta de Trabajo y Banda de Error de Una Desviación Estándar}

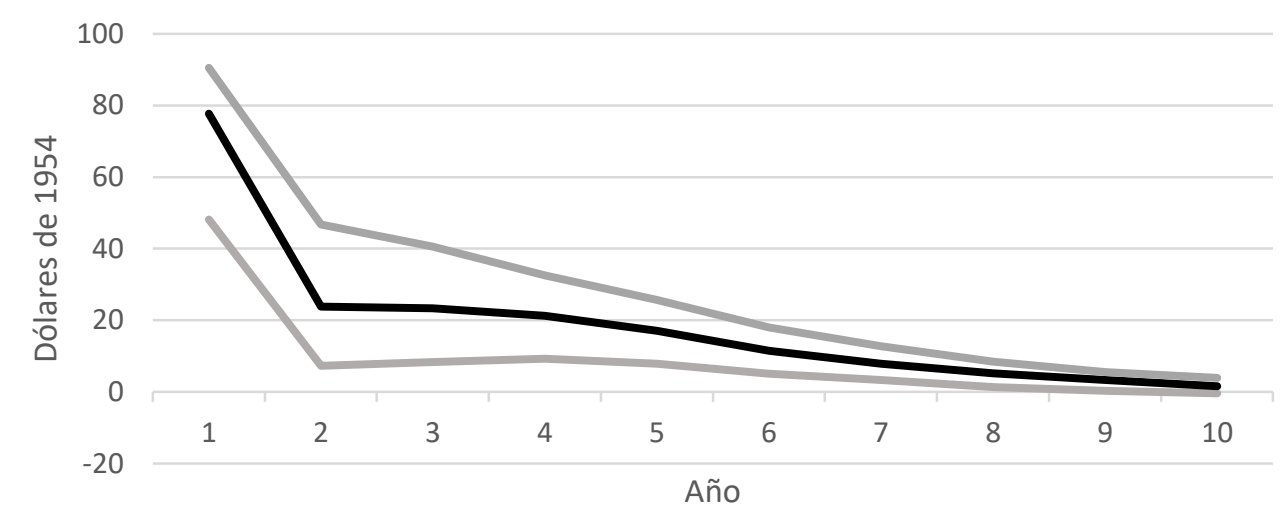

\begin{tabular}{|c|c|}
\hline $\begin{array}{l}\text { Cuadro 10 } \\
\text { Descomposición de la Varianza del Error de Predicción del Empleo } \\
\text { (Modelo SVAR) }\end{array}$ \\
\hline $\begin{array}{c}\text { Horizonte de } \\
\text { Predicción }\end{array}$ & $\begin{array}{c}\text { Porcentaje de la varianza atribuible a impulsos en la oferta } \\
\text { de trabajo }\end{array}$ \\
\hline \hline 1 & 34 \\
\hline 2 & 33 \\
\hline 3 & 33 \\
\hline 4 & 36 \\
\hline 8 & 39 \\
\hline 9 & 39 \\
\hline 10 & 39 \\
\hline
\end{tabular}

Por otra parte, a la luz de la información presentada en el Cuadro 10, los impulsos de la demanda de trabajo explican un poco más del 60 por ciento de las fluctuaciones en el empleo total de Puerto Rico en el corto y largo plazo. Así que, los impulsos de productividad parecen ser más importantes que los de las preferencias por ocio para la dinámica del insumo laboral en la isla. Ese es un resultado que se ha sido destacado en la literatura de los ciclos económicos reales (CER) siendo los trabajos pioneros Kydland 
y Prescott (1982 ), Long y Plosser (1983), King y Plosser (1984 ) y Long y Plosser (1987). En los modelos construidos de acuerdo con los preceptos de los CER, los choques de tecnología, que afectan la productividad marginal del trabajo, son las fuentes principales del ciclo económico.

Para el caso de Puerto Rico las perturbaciones de la demanda de trabajo pudieran estar asociados a las limitaciones en inversiones de capital en el área de manufactura que está experimentado la isla desde 2006, que han impactado la productividad del insumo laboral. La salida de empresas manufactureras estadounidenses de la isla, ante la perdida de incentivos de impuestos, pudiera ser una explicación para el rol prominente de este tipo de innovación en la dinámica del empleo en la isla (véase Toledo (2017)). Este punto se examina en el próximo apartado.

\subsection{Análisis de Robustez}

Para examinar la robustez de los resultados informados en la sección anterior con respecto a las variables consideradas y a la identificación de los impulsos estructurales se añadió al modelo SVAR la primera diferencia del capital $(\mathrm{K})$ y se impusieron restricciones de largo plazo. Este sistema permite evaluar los tres tipos de impulsos del modelo DSGE. Las restricciones de largo plazo son similares a las utilizadas por Galí (1999) ${ }^{6}$. El vector de las variables del modelo ampliado se puede representar como $\left[\begin{array}{lll}\Delta(W / P)_{t} & \Delta L_{t} & I_{t}\end{array}\right]^{\prime}$, donde $I_{t}\left(\Delta K_{t}\right)$ es la inversión total en términos reales ${ }^{7}$. Este modelo permite examinar el impacto de la perturbación que afecta la acumulación del capital, además de las otras ya examinadas, sobre el empleo. El vector de las perturbaciones esta dado por: $\left[\begin{array}{lll}\epsilon_{T e c, t} & \epsilon_{N T e c, t} & \epsilon_{I, t}\end{array}\right]^{\prime}$, donde, $\epsilon_{T e c, t}, \epsilon_{N t e c, t}, \mathrm{y} \epsilon_{I, t}$ son impulsos, de tecnología, no tecnológicos y los asociados a la inversión, respectivamente.

Para lograr, la identificación de los impulsos estructurales se imponen las siguientes restricciones (basadas en Galí (1999)): 1. En el largo plazo los salarios reales son impactados sólo por los impulsos de tecnología, 2. L se ve afectado permanentemente por las perturbaciones tecnológicas y no tecnológicas 3. Los tres tipos de shocks tienen

\footnotetext{
${ }^{6}$ Galí utiliza un sistema con la productividad del trabajo y las horas trabajadas. Francis y Ramey (2002) y Gamber y Joutz (1993) sustituyen la productividad del trabajo por el salario real.

${ }^{7}$ Fisher (2006) incluye el precio real de la inversión, en lugar de la primera diferencia de K, para identificar choques tecnológicos específicos asociados a la inversión, mientras que Watanabe (2012) utiliza la razón inversión-producción agregada real, basados en modelos teóricos diferentes al utilizado en esta investigación. Justiniano, Primicery y Tambalotti (2010) utilizan la inversión como en este artículo.
} 
efectos permanentes sobre la inversión. La primera restricción implica que los salarios reales se afectan permanente únicamente como resultado de cambios en la productividad proveniente de los impulsos tecnológicos. Así que, los impulsos tecnológicos son los únicos que afectan la productividad en el largo plazo. La segunda restricción permite que los impulsos no tecnológicos afecten el empleo en el largo plazo, pero no la productividad. Saltari y Travaglini (2009) asocian este tipo de perturbación a regulaciones institucionales que afectan la oferta de trabajo, como cambios en los beneficios marginales de los trabajadores o leyes de salario mínimo. Sin embargo, otros factores no relacionados con la oferta del insumo laboral que hayan afectado permanentemente al empleo están recogidos en ese shock. Para el caso de Puerto Rico, y otros países con limitaciones de capital, el gobierno es un motor importante en la creación y promoción de empleos, por lo que cabe la posibilidad de que dicha perturbación recoja los efectos de la política pública sobre la estructura económica. Esas dos primeras restricciones sólo permiten efectos transitorios ${ }^{8}$ de la inversión sobre las sobre L y $\frac{W}{P}$ y tienen como resultado que la raíz unitaria de L provenga de los impulsos idiosincráticos del mercado de trabajo.

La tercera restricción asegura que la inversión se vea afectada permanente por los impulsos de tecnológicos y no tecnológicos y es compatible con el modelo desarrollado por Hall (1997). El impulso de la inversión al no tener impacto permanente sobre el salario real ni el empleo se puede interpretar como una perturbación de la demanda agregada.

Es relevante señalar, que una porción importante de la inversión en Puerto Rico ha provenido de EE. UU. y depende, en parte, de las leyes de impuestos de ese país y cambios en la competitividad global, por lo que su permanencia siempre está en cuestionamiento, lo que justifica el que sus impactos sobre el salario real (un indicador de la productividad) y el empleo sean de carácter transitorio. Además, de 30 a 40 por ciento de la inversión en la isla es en la actividad de construcción que genera muchos puestos de trabajo temporeros. No obstante, se realizó una estimación permitiendo que los impulsos de la formación de capital tuvieran impactos permanentes sobre el empleo, y los hallazgos varían ligeramente, pero las conclusiones se mantienen.

\footnotetext{
${ }^{8}$ Esta identificación es similar a la utilizada por Saltari y Travaglini (2009), pero ellos utilizan el PIB en lugar de la inversión.
} 
El modelo se estimó con dos rezagos que es el orden óptimo determinado utilizando el criterio de Akaike. El mismo ajustó bien los datos y sus residuos constituyen procesos puramente aleatorios: el estadístico-Q multivariable de orden 5 fue de 31.00 y su Valor$\mathrm{P}$ igual a 0.27 , por lo que no existe evidencia de autocorrelación en los residuos hasta ese orden. El examen de las raíces del polinomio característico del sistema reveló que el mismo es estable.

El Cuadro 11 contiene la descomposición del error de predicción del empleo, bajo este nuevo modelo ampliado. Como se observa, los choques no tecnológicos, que incluyen cambios en la oferta de trabajo, explican más de 50\% de las variaciones de L en el corto plazo y de $47 \%$ en el largo plazo. El impacto de largo plazo es cuatro puntos porcentuales mayor al obtenido en la descomposición asintótica del modelo de equilibrio general que fue $43 \%$ y excede por ocho unidades el estimado del SVAR con restricciones de signos en ese horizonte de predicción. Sin embargo, en ese último modelo mencionado en el corto plazo las innovaciones de la oferta de trabajo explican $34 \%$ de las variaciones del empleo lo que representa una diferencia significativa al compararlo con el modelo ampliado. Esto pudiera implicar, como se mencionó antes, que esa perturbación incluye otras innovaciones que afectan L permanentemente, además de la de preferencia por ocio. Para el caso de Italia y Francia, Salteri y Travaglini (2009) encuentran que más de 75\% de la variancia del error de predicción del empleo es explicada por impulsos no tecnológicos.

Como es evidente en el Cuadro 11, los impulsos tecnológicos son responsables de cerca de 12 por ciento de las fluctuaciones en el empleo en el corto plazo. En el largo plazo, esas perturbaciones explican un poco más de un quinto de las desviaciones del insumo laboral de su tendencia de crecimiento. En el modelo de equilibrio general calibrado para Puerto Rico este tipo de shock explica el $26 \%$ de la variancia del error de predicción de L. Salteri y Travaglini (2009) hallan que los choques de tecnología explican entre 7\% y $15 \%$ de las variaciones en el insumo laboral en Italia y Francia, respectivamente. 


\begin{tabular}{|c|c|c|c|}
\hline \multicolumn{4}{|c|}{$\begin{array}{l}\text { Cuadro } 11 \\
\text { Descomposición de la Varianza del Error de Predicción del Empleo } \\
\text { (Modelo SVAR Ampliado) }\end{array}$} \\
\hline \multirow{2}{*}{$\begin{array}{l}\text { Horizonte } \\
\text { de } \\
\text { Predicción }\end{array}$} & \multicolumn{3}{|c|}{ Porcentaje de la varianza atribuible a impulsos: } \\
\hline & $\begin{array}{c}\text { De Tecnología } \\
\text { (Con efectos permanentes } \\
\text { sobre W/P) }\end{array}$ & $\begin{array}{l}\text { No Tecnológicos } \\
\text { (Con efectos transitorios } \\
\text { sobre W/P) }\end{array}$ & $\begin{array}{c}\text { De la } \\
\text { Inversión }\end{array}$ \\
\hline 1 & 13 & 56 & 31 \\
\hline 2 & 11 & 57 & 32 \\
\hline 3 & 19 & 52 & 29 \\
\hline 4 & 20 & 51 & 29 \\
\hline 8 & 21 & 47 & 32 \\
\hline 9 & 21 & 47 & 32 \\
\hline 10 & 21 & 47 & 32 \\
\hline \multicolumn{4}{|c|}{ Fuente: Elaboración propia } \\
\hline
\end{tabular}

Las innovaciones de inversión, por su parte, dan cuenta de cerca de un tercio de las fluctuaciones del empleo de su tendencia de largo plazo. Este resultado concuerda con Rodríguez (2018) quien usando datos mensuales para el período de 1976 al 2010, estima que los choques del lado de la demanda (externos y locales) son responsables de cerca del 35\% de la varianza del error de predicción del desempleo en Puerro Rico. Para el caso de EE. UU. Justiniano, Primiceri y Tambalotti (2010), utilizando datos trimestrales de 1954 a 2004, hallan que los impulsos de inversión explican entre 20 y $60 \%$ de las variaciones en el insumo laboral en la frecuencia del ciclo económico (entre 1.5 a 8 años). Por otra parte, un análisis que realiza Fisher (2006) con datos de 1955 al 2000 de ese mismo país, indica que entre 25 y $59 \%$ de la varianza del error de predicción de las horas trabajadas son el resultado de los shocks de la inversión.

Para examinar cómo los tres tipos de impulsos han afectado la serie de empleo a través del tiempo se utiliza la descomposición histórica de la serie de empleo para el período de 1953 a 2019. Ese tipo de análisis provee una interpretación de las fluctuaciones de la variable analizada tomando como base los impulsos estructurales identificados. La representación MA del modelo SVAR (VMA) implica que cada una de las series del modelo se puede expresar como una combinación lineal de los choques que la han 
impactado en el pasado, tres en este caso. Por tanto, es posible evaluar lo que hubiera sucedido si sólo uno de los impulsos hubiera actuado sobre la serie bajo análisis. Es como si se apagaran los efectos de dos de las perturbaciones a la vez y se dejara sólo uno encendido. Entonces es posible comparar las fluctuaciones que son generadas por el shock que se dejó encendido sobre el empleo, en este caso, con las que sucedieron. Haciendo ese ejercicio para cada de los tres tipos de impulsos, se puede determinar en cada período cuál de los impulsos tuvo un impacto mayor sobre la variable analizada. La representación de estas descomposiciones para distintos subperíodos se presenta en las Gráficas 5, 6, y 7. La partición de las series se realizó para facilitar la interpretación del análisis y no refleja necesariamente una periodización de la historia económica de Puerto Rico.

En el primer subperíodo examinado, de 1953 a 1970, los impulsos no tecnológicos son los que mejor explican las desviaciones del empleo de su tendencia de crecimiento, como se muestra en la Gráfica 5. Los choques de inversión también reproducen bastante bien el movimiento general de las fluctuaciones de la serie examinada, pero no explican mucho de las reducciones en las mismas. En ese período, de la industrialización de la isla, los avances tecnológicos parecen tener su mayor poder explicativo sobre las variaciones de L de 1956 a 1966.

Las perturbaciones tecnológicas, durante los años de 1971 a 1999, en términos generales, se mueven en la misma dirección que L. Sin embargo, su capacidad predictiva es baja en el ciclo económico, como se aprecia en la Gráfica 6. Se observa, por ejemplo, que los períodos recesionarios de los mil novecientos setenta, ochenta y noventa no parecen ser el resultado de ese tipo de impulso. Por otro lado, las innovaciones en la inversión real explican tanto el movimiento general de la serie como una proporción alta las fluctuaciones cíclicas de 1971 hasta 1994, antes de la eliminación de los incentivos de impuestos de las empresas estadounidense (conocida como la sección 936 del código de rentas internas de EE. UU., cuyo cese comenzó en 1996 y finalizó en el 2006). Se puede observar que en la recesión de los $1980^{9}$, que se plantea fue causada por la política monetaria restrictiva de Estados Unidos que incrementó considerablemente las tasas de interés, los impulsos de la inversión son los que mejor explican las reducciones en el

\footnotetext{
${ }^{9}$ Conocida como la recesión Reagan (en referencia al Presidente de Estados Unidos en ese momento, Ronald Reagan) o la recesión Volcker refiriéndose a Paul Adolph Volcker presidente del banco central de EE. UU., la Reserva Federal, de 1979 a 1987.
} 
nivel de empleo. Es evidente, en la Gráfica 6, que los impulsos no tecnológicos también juegan un rol relevante en la dinámica del empleo de Puerto Rico durante ese período. En la recesión de los setenta, ese último tipo de perturbación mencionada explica una proporción mayor de las fluctuaciones en el insumo laboral que las asociadas a la inversión y lo mismo sucede en la expansión del último lustro del siglo 20.

La descomposición histórica del empleo para la última subdivisión del período utilizado en la estimación del modelo, 2000 a 2019, se encuentra en la Gráfica 7. Los impulsos de tecnología identificadas en el modelo son los más que parecen haber influenciado la serie bajo análisis entre 2006 y 2015: el período luego del cese de los incentivos de impuestos para las firmas de EE. UU. bajo la sección 936. La reducción en el empleo de manufactura, producto de la salida de algunas empresas intensivas en capital puede ser una explicación para este resultado. Los choques no tecnológicos, por su parte, explican una proporción significativa de las desviaciones del empleo del 2000 al 2007, y de 2013 hasta 2017. Los impulsos de inversión, por otro lado, explican las fluctuaciones del empleo del 2005 al 2012. Durante esos años esas innovaciones son capaces de explicar una parte considerable de las bajas en los niveles del empleo. Es evidente en la Gráfica 7, que luego del impacto del huracán María sobre la isla (2017), las innovaciones en la inversión son las que se mueven en la misma dirección que las oscilaciones del empleo. Estas innovaciones, no obstante, predicen un nivel mayor del empleo que el que sucedió. Esa cifra de empleo tal vez hubiera sido posible sin la devastación de la infraestructura que experimentó el país como consecuencia del huracán mencionado.

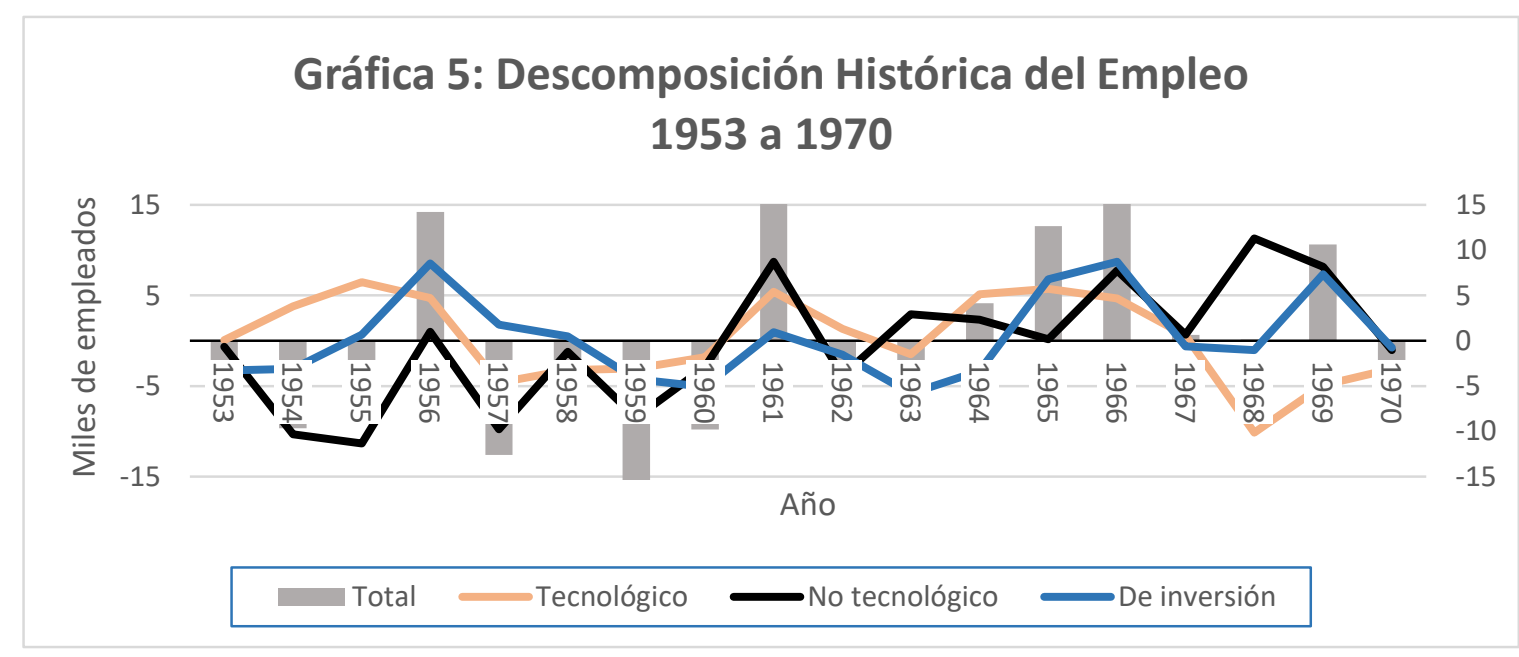




\section{Gráfica 6: Descomposición Histórica de la Serie de Empleo} 1971 a 1999
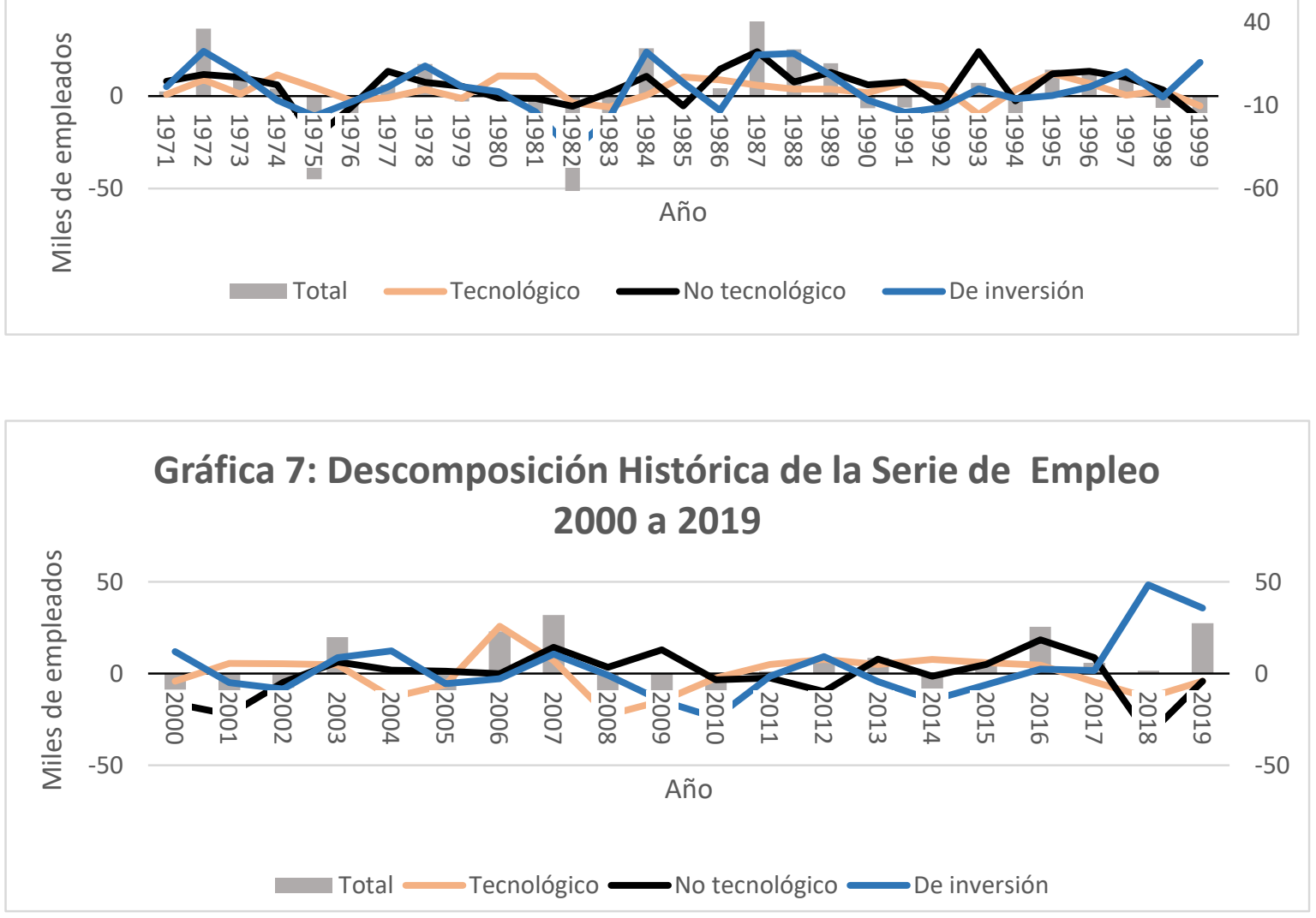

\section{CONCLUSIONES}

Este artículo tuvo como propósito comparar los efectos de impulsos de la oferta y demanda de trabajo sobre la trayectoria del empleo agregado en Puerto Rico. Se calibró un modelo de equilibrio general de ciclos económicos reales utilizando valores para los parámetros lo más cercanos posible a los implícitos en las series que describían el estado de la economía de la isla en el último lustro. Los resultados de la simulación de ese modelo revelaron que los impulsos de las preferencias por ocio pueden tener un efecto importante en las fluctuaciones del insumo laboral. Además, se estimaron dos modelos SVAR utilizando restricciones de signos en uno de ellos y la descomposición BlanchardQuah en el otro, para identificar perturbaciones de la demanda y oferta de trabajo que han afectado la serie de empleo y otras variables del país para el período de 1950 a 2019. Los resultados de la estimación de esos modelos sugieren que los impulsos de la demanda de 
trabajo (productividad y/o choques de la inversión) se les puede atribuir entre cerca del 50 y $70 \%$ de las fluctuaciones que ha experimentado el empleo en la isla. También implican que los impulsos de la oferta de trabajo son responsables de al menos $30 \%$ de las desviaciones del insumo laboral de su tendencia de largo plazo. No obstante, la importancia de los tres tipos de perturbaciones en la dinámica del empleo agregado varió para diferentes períodos. De 1953 a 1970, los impulsos no tecnológicos (que incluyen los de la oferta de trabajo) fueron los que mejor explicaban las desviaciones de ese indicador macroeconómico de su tendencia de crecimiento. En el período de 1971 a 1999 a las innovaciones de la inversión se les pueden atribuir una proporción alta tanto del movimiento general de la serie de empleo como de sus fluctuaciones cíclicas, pero los cambios no anticipados en la oferta laboral (choques no tecnológicos) también tienen una alta contribución en esa dinámica. En años más recientes, entre 2006 y 2015, los impulsos de tecnología son los que parecen haber impactado más la serie bajo análisis, mientras que los choques no tecnológicos, explican una proporción significativa de las desviaciones del empleo del 2000 al 2007 y de 2013 hasta 2017. Por su parte, los cambios inesperados en la inversión son los que explican mejor los períodos de reducciones en el empleo en las primeras dos décadas del siglo 21.

Los hallazgos que se obtuvieron en esta investigación sobre la importancia de los choques de la demanda de trabajo y la inversión sobre las fluctuaciones del empleo, en una economía con limitaciones de capital como la de Puerto Rico, no son sorprendentes. No obstante, la aportación de los impulsos de la oferta a esas variaciones no era un hallazgo anticipado, por lo que merece algunas reflexiones. Esos impulsos del lado de la oferta laboral pudieran estar asociados a tres categorías, no necesariamente mutuamente excluyentes, a saber, cambios en: las preferencias por ocio, en la productividad de actividades sin remuneración o en la brecha entre el salario y la tasa de sustitución de consumo por ocio. Algunas fuentes de los impulsos para esas categorías son los beneficios de desempleo, pagos de transferencias públicas y privadas, cambios en las regulaciones laborales y leyes de salario mínimo entre otras (véase Foroni et al. (2016)). Por ejemplo, si ocurre una reducción en los beneficios marginales de los empleados, disminuye el costo del ocio lo que impacta las decisiones sobre la selección consumo/ocio que toman los individuos, lo que puede ocasionar que algunos individuos salgan de la fuerza trabajadora, tal vez, acogiéndose a la jubilación temprana. De igual 
forma pudiera implicar que ciertos miembros de los hogares (trabajadores secundarios) se dediquen a tareas tales como estudiar o participar en actividades de organizaciones sin fines de lucro, lo que mermaría las horas dedicadas a trabajar o buscar empleo en la economía. Además, esas bajas en los beneficios marginales de los empleados afectan la diferencia entre la compensación recibida por el trabajo (W) y la tasa de sustitución de ocio por consumo $\left(\mathrm{MRS}_{\text {ocio/consumo }}\right.$ ) o las unidades de ocio que los individuos están dispuestos a ceder por una unidad adicional de consumo. Si el incremento en la utilidad que los individuos recibirían por el alza en consumo que pueden obtener del ingreso que proviene del salario de una hora adicional de trabajo no compensa por la pérdida de utilidad asociada a la reducción en el tiempo de ocio, las personas no se verán motivados a incrementar sus horas de trabajo. Obsérvese que la $\mathrm{MRS}_{\text {ocio/consumo depende de la }}$ valoración que hagan los individuos sobre el consumo y el ocio. La etapa del ciclo de vida en que las personas se encuentren afecta esa tasa de sustitución, por lo que factores

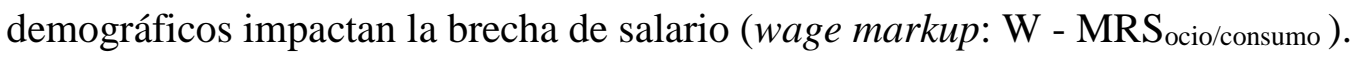

Así que, estímulos a la oferta de ese insumo de producción, aun en economías con alto nivel de desempleo, pudiera tener efectos en el nivel del empleo. Por ejemplo, pudiera ser que algunos sectores económicos que utilizan mano de obra semicualificada y no calificada tengan puestos de trabajo vacantes y que les resulte dificultoso la contratación de los recursos idóneos, por lo que ese tipo de medida pudiera tener, al menos, un impacto marginal sobre la trayectoria del empleo. Por tanto, aunque los análisis realizados sugieren que el diseño de políticas públicas dirigidas a promover la creación de puestos de empleo tendría el impacto mayor sobre la dinámica del empleo en el país, como se ha planteado anteriormente (véase por ejemplo Liard-Muriente y Schenck (2020)), el impacto de la participación laboral en el nivel de empleo no parece ser tan insignificante como para que se pueda descartar. Así que, medidas dirigidas a mejorar las condiciones de empleo de los trabajadores pudieran ser efectivas para promover la actividad económica en la isla. Estas políticas del mercado laboral pudieren incidir sobre la productividad del trabajo y las preferencias sobre consumo y ocio, dos factores que de acuerdo con Bergstrom y Dodds (2021) afectan la desigualdad de ingresos en los Estados Unidos.

Es menester señalar que este estudió tuvo la limitación principal de que no se contó con información sobre las horas de trabajo y en su lugar se utilizó el empleo como la variable 
principal del análisis en los modelos SVAR. Medir el insumo laboral con el número de empleados tiene la dificultad de que esa variable pudiera aumentar, aunque el total de horas trabajadas no se incremente, por el efecto de la sustitución de trabajadores a tiempo parcial por los que laboran a jornada completa. Por tanto, los resultados de la estimación pudieran variar al utilizar las horas trabajadas en lugar del empleo. Sin embargo, como los hallazgos de este trabajo son compatibles con otros de la literatura sobre el tema y lo encontrado en el modelo de equilibrio general calibrado para Puerto Rico, es de esperarse que en términos cualitativos los hallazgos se mantengan.

\section{REFERENCIAS BIBLIOGRAFICAS}

Aaronson S., Cajner T., Fallick B., Galbis-Reig F., Smith C. y Wascher W. (2014). Labor Force Participation: Recent Developments and Future Prospects. Brookings Papers on Economic Activity, 45(2): 197-275.

Alemar, E. A. y Rodríguez-Ramos, C.A. (2020). Efectos Reales de Impulsos Tecnológicos: El Caso de Puerto Rico. Estudios Económicos, aparecerá próximamente.

Autor, D. H. (2007). Structural demand shifts and potential labor supply responses in the new century. Federal Reserve Bank of Boston, Monografía, 52.

Basu, S., Fernald J. G. y Kimball M. S. (2006). Are Technology Improvements Contractionary? American Economic Review, 96 (5): 1418-1448.

Bergstrom, K. y Dodds, W. (2021). Using Labor Supply Elasticities to Learn about Income Inequality: The Role of Productivities versus Preferences, American Economic Journal: Economic Policy, 13 (3): 28-62.

Blanchard O. J. y Quah D. (1989). The Dynamic Effects of Aggregate Demand and Supply Disturbances. The American Economic Review,79(4): 655-73.

Blanchard O. J. y Summers L. H. (1986). Hysteresis and the European Unemployment Problem. Massachusetts Institute of Technology (MIT), Departamento de Economía, Documento de Trabajo 427.

Braun, H., Bock, R. De, y Dicecio, R. (2009). Supply Shocks, Demand Shocks, and Labor Market Fluctuations. Federal Reserve Bank of St. Louis Review, 91(3): 155-178. Canova, F., y De Nicoló G. (2002). Monetary Disturbances Matter for Business Fluctuations in the G-7. Journal of Monetary Economics, 49(6): 1131-1159. 
Chang Y. y and Schorfheide, F. (2003). Labor-Supply Shifts and Economic Fluctuations. Journal of Monetary Economics, 50(8): 1751-1768.

Christiano, LJ, Eichenbaum, M. y Vigfusson, R. (2003). What Happens after a Technology Shock? SSRN 425004. https://doi.org/10.2139/ssrn.425004

Clark, K. B, y Summers L. H. (1982) Labor Force Participation: Timing and Persistence. The Review of Economic Studies, 49(5): 825-844, https://doi.org/10.2307/2297190

Collard F. (2009) Stochastic simulations with DYNARE. A practical guide. (GREMAQ, University of Toulouse) https://www.coursehero.com/file/11408329/guide

Erceg, C. y Levin, A. (2014). Labor Force Participation and Monetary Policy in the Wake of the Great Recession. Journal of Money, Credit and Banking, 46(2): 3-49.

Faust, J (1998). The Robustness of Identified VAR Conclusions about Money. CarnegieRochester Conference Series on Public Policy, 49: 207-44.

Fisher, J. D. M., ( 2006). The Dynamic Effects of Neutral and Investment-Specific Technology Shocks. Journal of Political Economy, 114(3): 413-451.

Foroni, C., Furlanetto, F.y Lepetit, A. (2016). Labour Supply Factors and Economic Fluctuations, Norges Bank, Oslo, Working Paper, No. 07/2015, ISBN978-827553-864-0, http://hdl.handle.net/11250/2495867

Francis B. y Ramsey, V. (2002), "Is the Technology-Driven Real Business Cycle Hypothesis Dead? Shocks and Aggregate Fluctuations Resisted”, National Bureau of Economic Research, Working Paper 8726.

Galí J. (1999), “Technology, Employment, and Business Cycle: Do Technology Shocks Explain Aggregate Fluctuations? The American Economic Review, 89(1), 249271.

Gamber, E. N. y Joutz, F. L, (1993). The Dynamic Effects of Aggregate Demand and Supply Disturbances: Comment. American Economic Review, 83(5): 1387-1393. Goettle, R. J, Ho M. S., Slesnick, D. 1. T., Wilcoxen, P. J, y Jorgenson, D. (2008).U.S. Labor Supply and Demand in the Long Run. Journal of Policy Modeling, 30(4): 603-618.

Hall, R. E., (1997). Macroeconomic Fluctuations and the Allocation of Time. Journal of Labor Economics, University of Chicago Press, vol. 15(1), pages 223-250. 
Justiniano, A., Primiceri, G.E., y Tambalotti, A. (2010). Investment shocks and business cycles, Journal of Monetary Economics, 57(2): 132-145.

King, R., G., y Plosser C. (1994). Money, Credit, and Prices in a Real Business Cycle. American Economic Review, 74(3): 363-380.

Kydland, F. E. and Prescott, E.C. (1982). Time to Build and Aggregate Fluctuations. Econometrica, 50(6): 1345-70.

Liard-Muriente C. y Schenck, S.M (2020). Tax Incentives to Promote Small Firms in Puerto Rico, Pennsylvania Economic Review, 27(1): 44-53.

Long, J. B, Jr y Plosser, C. I. (1987). Sectoral vs. Aggregate Shocks in the Business Cycle. American Economic Review, American Economic Association, 77(2): 333-336.

Long, J. B, Jr Plosser, C. (1983). Real Business Cycles. Journal of Political Economy, 91(1): 39-69.

MacKinnon, J.G., Haug, A.A. and Michelis, L. (1999) Numerical Distribution Functions of Likelihood Ratio Tests for Cointegration. Journal of Applied Econometrics, 14(5): 563-577.

McCallum B. T. (1988). Real Business Cycle Models, National Bureau of Economic Research, Documento de Trabajo 2480.

Pesavento, E. y Rossi, B. (2003). "Do Technology Shocks Drive Hours Up or Down? A Little Evidence from Agnostic Procedure", Manuscrito, Departamento de Economía Emory University.

Rodríguez, Carlos A. (2018). Fuentes de las Fluctuaciones Macroeconómicas en Puerto Rico. Estudios Económicos, 33(2): 219-252.

Saltari, E. y Travaglini G. (2009). The Productivity Slowdown Puzzle. Technological and Non-technological Shocks in the Labor Market. International Economic Journal, 23(4): 483-509, DOI: 10.1080/10168730903377819

Shapiro M. y Watson M. (1988). Sources of Business Cycles Fluctuations. NBER Macroeconomics Annual, 3: 111-156.

Shea, J. (1999). What Do Technology Shocks Do? en B. Bernanke y J. Rotenberg, eds., NBER Macroeconomics Annual, Cambridge, MA: MIT Press.

Smets, F. y Wouters, R. (2007). Shocks and Frictions in US Business Cycles: A Batesian Approach. American Economic Review, 97(3): 586-606. 
Summers Lawrence H. (1986). Why is the Unemployment Rate So Very High near Full Employment. Brookings Papers on Economic Activity, 17(2): 339-396.

Toledo, W. (2006). Avances Tecnológicos y las Fluctuaciones Económicas: Evidencia del Sector Manufactura de Puerto Rico. Econoquantum, 3(1):35-61

Toledo, W. (2017) Foreign Direct Investment and Manufacturing Growth: The Case of Tax Incentives in Puerto Rico. Modern Economy, 8, 272-281. doi: 10.4236/me.2017.82019.

Uhlig H. (2003). Do Technology Shocks lead to a fall in total hours worked? Manuscrito, Humboldt University Berlin.

Uhlig, H. (2005). "What Are the Effects of Monetary Policy on Output? Results from an Agnostic Identification Procedure.” Journal of Monetary Economics, 52(2): 381419.

Watanabe, S. (2012). The Role of Technology and Nontechnology Shocks in Business Cycles. International Economic Review, 53(4): 1287-1321. 